\title{
A note on an identity of Jacobi's
}

\author{
By Nancy Walls.
}

That the determinant

$\left|\begin{array}{lccc}h_{r} & h_{s} & \cdots & h_{t} \\ h_{r-1} & h_{s-1} & \ldots & h_{t-1} \\ h_{r-2} & h_{s-2} & \ldots & h_{t-2} \\ \ldots \ldots \ldots & \ldots \ldots \ldots \ldots \ldots \ldots \ldots \ldots\end{array}\right|$.

where $h_{r}$ is the $r^{\text {th }}$ complete homogeneous symmetric function in a set of $n$ arguments, is equal to the quotient of a particular pair of alternants was shown essentially by Jacobi in 1841 and by Trudi in 1864. The present note exhibits this well-known relation, (3), as the immediate consequence of a simple matrix equality.

The symmetric functions $h_{r}$ are connected with the elementary symmetric functions $a_{r}$ in the same $n$ arguments $a, \beta, \ldots, \kappa$ by the Wronski relations

$$
\begin{aligned}
a_{0} h_{1}-a_{1} h_{0} & =0, \\
a_{0} h_{2}-a_{1} h_{1}+a_{2} h_{0} & =0, \\
a_{0} h_{3}-a_{1} h_{2}+a_{2} h_{1}-a_{3} h_{0} & =0,
\end{aligned}
$$

obtained by equating the coefficients of powers of $x$ in the identity

where

$$
\mathcal{H}(\alpha, \beta, \ldots, \kappa ; x) \mathcal{H}(\alpha, \beta, \ldots, \kappa ; x)=1 \text {, }
$$

and

$$
\mathscr{H} \equiv(1-\alpha x)(1-\beta x) \ldots(1-\kappa x)
$$

$\mathcal{H} \equiv\left(1+\alpha x+\alpha^{2} x^{2}+\ldots\right)\left(1+\beta x+\beta^{2} x^{2}+\ldots\right) . .\left(1+\kappa x+\kappa^{2} x^{2}+\ldots\right)$

are the generating functions of the $a_{r}$ and the $h_{r}$ respectively. Let us similarly equate coefficients in the identity

$\mathcal{A}(\beta, \gamma, \ldots, \kappa ; x) \mathcal{H}(a, \beta, \gamma, \ldots, \kappa ; x)=1+\alpha x+a^{2} x^{2}+\ldots$

We obtain relations of the type

$$
\text { (a) } a_{0} h_{r}-(a) a_{1} h_{r-1}+(\alpha) a_{2} h_{r-2}-\ldots=a^{r},
$$

where $(a) a_{r}$ denotes the $r^{t h}$ elementary symmetric function in the arguments with $a$ omitted, a set of $n-1$ arguments, so that all $a_{r}=0$ for $r \geqq n$. 
In virtue of such relations for the different arguments we have

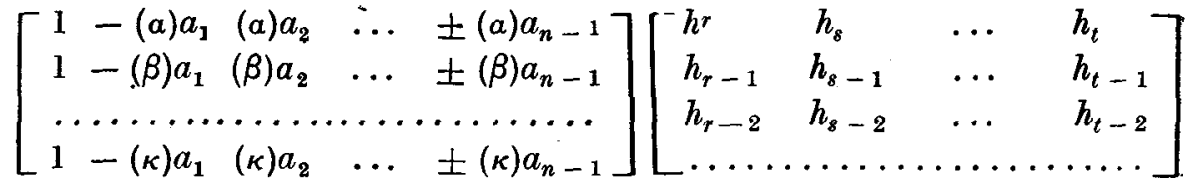

$$
\begin{aligned}
& =\left[\begin{array}{cccc}
a^{r} & a^{s} & \ldots & a^{t} \\
\beta^{r} & \beta^{s} & \ldots & \beta^{t} \\
\ldots \ldots & \ldots & \ldots & \ldots \\
\kappa^{r} & \kappa^{s} & \ldots & \kappa^{t}
\end{array}\right] .
\end{aligned}
$$

If we take as second factor on the left hand side the matrix

$$
H \equiv\left[\begin{array}{ccccc}
h_{0} & h_{1} & h_{2} & \ldots & h_{n-1} \\
\bullet & h_{0} & h_{1} & \ldots & h_{n-2} \\
\cdots & \ldots & \ldots & \ldots & \ldots \\
\bullet & \bullet & \bullet & \cdots & h_{0}
\end{array}\right],
$$

so that $|H|=1$, the corresponding determinantal relation gives us

$$
\left|1-(\beta) a_{1} \quad(\gamma) a_{2} \quad \ldots \quad \pm(\kappa) a_{n-1}\right|=\left|\begin{array}{lllll}
a^{0} & \beta^{1} & \gamma^{2} & \ldots & \kappa^{n-1}
\end{array}\right|,
$$

as is otherwise obvious from consideration of the linear factors of each determinant, and hence the determinantal form of (2) is equivalent to

$$
\left|\boldsymbol{a}^{0} \beta^{1} \gamma^{2} \ldots \kappa^{n-1}\right|\left|h_{0} h_{r-1} h_{\delta-2} \ldots h_{t-n+1}\right|=\left|\boldsymbol{a}^{0} \beta^{r} \gamma^{s} \ldots \kappa^{t}\right|,
$$

Jacobi's identity.

It may be remarked in passing that, since the reciprocal of $H$ is

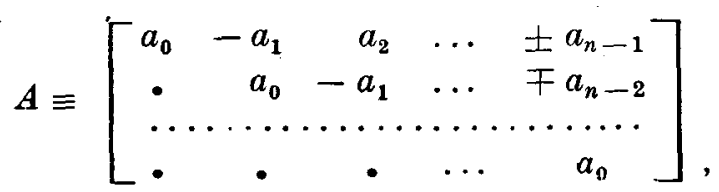

because of the Wronski relations, the particular form of (2) with $r, s, \ldots, \mathrm{t}=0,1, \ldots, n-1$ may also be written

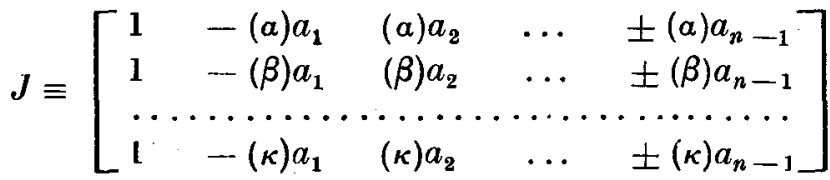

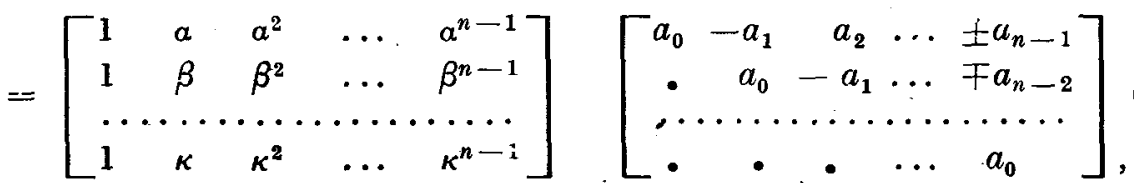

the determinantal form of which has been noted by Muir (Theory of 
Determinants, IV, p. 148) in referring to a paper in which $(-)^{\frac{1}{3}(n-1)}:$ arises as the Jacobian of the functions $a_{1}, a_{2}, \ldots, a_{n}$.

The identity (1) may be extended to

$$
\mathcal{H}(\ldots ; x) \mathcal{H}(\ldots, \alpha, \beta, \ldots ; x)=\mathcal{H}(\alpha, \beta, \ldots ; x) .
$$

and similarly we have

$$
\mathcal{H}(\ldots, \alpha, \beta, \ldots ; x) \mathcal{H}(\ldots ; x)=\mathcal{H}\left(\alpha, \beta, \ldots ; x_{\downarrow}\right) .
$$

[In particular, (4) is an immediate consequence of

$$
\mathscr{H}(\alpha, \beta, \ldots, \kappa ; x) \mathcal{H}(\alpha ; x)=\mathscr{H}(\beta, \ldots, \kappa ; x) .]
$$

Further, $\mathscr{H}(\ldots, a, \beta, \ldots ; x) \mathcal{H}(\ldots, \lambda, \mu, \ldots ; x)$

$$
=\mathcal{H}(\alpha, \beta, \ldots ; x) \mathcal{H}(\lambda, \mu, \ldots ; x) .
$$

Generalizations of (2) may hence be obtained.

University College,

SoUthampton.

\section{A note on the "probleme des rencontres."}

\section{By A. C. Althen.}

1. This celebrated problem is treated in nearly all the textbooks on probability; for example in Bertrand's Calcul des.Probabilités, 1889, pp. 15-17, in Poincaré's of the same title, 1896, pp. 36-38, and in most of the recent textbooks. The problem may be stated in abstract terms as follows: Among the $n$ ! permutations $\left(\alpha_{1} \alpha_{2} \alpha_{3} \ldots a_{n}\right)$ of the natural order $(123 \ldots n)$, how many have no $\alpha_{j}$ equal to $j$ ? The problem has been clothed in many picturesque (and highly unlikely) "representations"; for example, by imagining $n$ letters placed at random in $n$ addressed envelopes, and inquiring what is the chance that no letter is in its correct envelope; or by imagining $n$ gentlemen returning at random to their $n$ houses; and so on, ad risum. Various derivations have also been given of the probability in question, namely

$$
p(0 ; n)=1-\frac{1}{1 !}+\frac{1}{2 !}-\frac{1}{3 !}+\ldots+(-)^{n} \frac{1}{n !}
$$

